\title{
CORRECTIONS
}

\section{Paediatric hearing loss}

\section{0-Minute Consultation: Paediatric hearing loss}

In this article by Hannah Nieto and colleagues (BMJ

2017;356:j803 doi:10.1136/bmj.j803), the guidance on diagnosis of sensorineural hearing loss requires clarification. The sentence: "If there is suspicion of syndromic causes, refer to a specialist such as a paediatrician, or otherwise an audiovestibular physician," should have read: "If there is suspicion of syndromic causes, refer to a paediatrician." 